\title{
Random Polyelectrolytes and Polyampholytes in Solution
}

\author{
Itamar Borukhov and David Andelman \\ School of Physics and Astronomy, Raymond and Beverly Sackler Faculty of Exact Sciences \\ Tel-Aviv University, Ramat-Aviv 69978, Tel-Aviv, Israel \\ Henri Orland \\ Service de Physique Théorique, CE-Saclay, 91191 Gif-sur-Yvette Cedex, France
}

(September 7, 2018)

\begin{abstract}
The behavior of polyelectrolytes and polyampholytes in semi-dilute solutions is investigated theoretically. Various statistical charge distributions along the polyelectrolyte chains are considered: (i) smeared, where the charges are uniformly distributed along the chain. (ii) Annealed, where the charges are allowed to associate and dissociate from the chain. (iii) Permuted, where the total number of charges on the chain is fixed, but the charges can move along the chain. (iv) Quenched, where the charges on the chains are "frozen" in a random configuration. Finally, we also consider (v) polyampholytes, where each monomer can be positively or negatively charged, or neutral. Path integral formulation was used to derive mean field free energies for the different models. Selfconsistent field equation is obtained for the polymer order parameter and a Poisson-Boltzmann like equation for the electrostatic potential. We show that the difference between the permuted and the smeared models is a constant shift in the chemical potential leading to similar mean field equations. Within mean-field the quenched model is found to be equivalent to the annealed one, provided that the system is coupled to a reservoir of polyelectrolyte chains. The random phase approximation is used to calculate the monomer-monomer structure factor $S(q)$ for the different statistical charge distribution models. We show that in the annealed model, fluctuations of the the monomer charges contribute to the electrostatic screening in addition to the free ions in the solution. The strength of this screening depends on the variance of the monomer charge distribution and is especially important for polyampholytes in bad solvent conditions where the mesophase separation is enhanced. The ratio between the variance and the net average charge determines whether polyampholytes behave as polyelectrolytes or as neutral chains.
\end{abstract}

61.25.H, 36.20, 41.10D

\section{INTRODUCTION}

Charged polymers have drawn a considerable amount of attention in the past years both theoretically and experimentally [1] 3]. This is due, on one hand, to their wide range of industrial applications in processes involving charged colloids 4.5 . and on the other hand, to their resemblance to water soluble bio-polymers such as proteins and DNA. A distinction is made between polyelectrolytes, with all charges having the same sign, and polyampholytes with positively as well as negatively charged monomers $\sqrt{6}-10]$. For the former, the electrostatic interactions are repulsive and long ranged. For the latter, the repulsion of like-charges competes with the attraction of opposite charges, resulting in a complex behavior which depends on the net charge of the chain.

In spite of extensive investigations of polyelectrolytes including the pioneering works of Katchalsky et al. [11] and Manning [12], polyelectrolytes are much less understood than neutral polymers. For example, there exists a debate regarding the persistence length and the chain conformations for single chains in dilute solutions 13 18]. This is due to the delicate interplay between the chain connectivity and the long range nature of electrostatic interactions.

Semi-dilute polyelectrolyte solutions where the chains interact with one another have been studied as well [16, 17, 19, 20]. In the so-called "blob" picture, scaling laws are derived by singling out the most dominant interaction at different length scales. Another technique is the Random Phase Approximation (RPA) [21 26] used for the calculation of the structure factor $S(q)$ as can be measured by scattering experiments [27, 28].

In addition to bulk properties, special attention was directed to adsorption experiments of polyelectrolytes onto a single charged surface [29 32], and between two charged surfaces 33 36]. On the theoretical side, discrete models have been employed for which the chains are placed on a lattice [37 40]. This approach has been used by Böhmer et al. [11] to calculate force curves between two charged surfaces containing a polyelectrolyte solution.

Another approach is a continuum one [42 46] where the charge densities, monomer densities and electric field are treated as continuous functions of the local position. Varoqui et al. 443, 144 investigated polyelectrolyte adsorption onto one surface, while Podgornik [47] calculated inter-surface forces. In recent works [4] non-linear excluded volume 
interactions have been included and scaling laws characterizing the adsorption of polyelectrolytes are proposed (within mean-field approximation).

In the present work the continuum approach is extended to random (heterogeneous) polyelectrolytes. We study several statistical charge distributions (i.e. annealed, quenched and permuted) corresponding to different physical situations. Polyampholytes with positive and negative charges are studied as well. One of the goals of the present work is to take explicitly into account several characteristics of polyelectrolytes such as the connectivity of the polymer chains, the non-electrostatic monomer-monomer interactions and the Coulomb interactions between charged monomers, counter-ions and co-ions.

The paper is organized as follows: in the next section we present a general formalism for calculating the free energy of randomly charged chains. This formalism is applied in Sec. III to derive mean-field equations for the various charge distributions (including polyampholytes). The reader who is not interested in the technical details can skip these two sections and go directly to Sec. IV where the mean-field results are summarized and the various charge distributions are compared. The random phase approximation (RPA) is used in Sec. V to calculate the structure factor $S(q)$ of polyelectrolytes and polyampholytes in good and bad solvents.

\section{GENERAL FORMALISM}

Let us consider a semi-dilute solution of polyelectrolytes in a good solvent in presence of salt (electrolyte). The system is schematically drawn on Fig. 1. In the model, based on the Edwards' formalism [48], the microscopic degrees of freedom are the monomer positions $\left\{\mathbf{r}_{l}(s)\right\}$, where $s \in[0, N]$ is a continuous index along a chain of $N$ monomers and $l=1, \ldots, M$ is the label of the $M$ chains in solution. The positions of the small co-ions (counter-ions) are denoted by $\mathbf{R}_{i}^{+}\left(\mathbf{R}_{j}^{-}\right)$where $i=1, \ldots, N^{+}$and $j=1, \ldots, N^{-}$are, respectively, the indices of the monovalent positive $(+e)$ and negative $(-e)$ ions (see Fig. 1). For simplicity only a symmetric 1:1 electrolyte is considered.

The partition function $Z$ is then expressed as a path integral over all possible configurations:

$$
\begin{aligned}
Z & =\int \mathcal{D} \mathbf{r}_{l}(s) \mathcal{D} \mathbf{R}_{i}^{+} \mathcal{D} \mathbf{R}_{j}^{-} \exp \left(-\frac{3}{2 a^{2}} \sum_{l=1}^{M} \int_{0}^{N} \mathrm{~d} s \dot{\mathbf{r}}_{l}^{2}(s)\right) \\
& \times \exp \left(-\frac{1}{2} \beta \int \mathrm{d} \mathbf{r} \mathrm{d} \mathbf{r}^{\prime} \hat{\rho}_{\mathrm{c}}(\mathbf{r}) v_{\mathrm{c}}\left(\mathbf{r}-\mathbf{r}^{\prime}\right) \hat{\rho}_{\mathrm{c}}\left(\mathbf{r}^{\prime}\right)-\frac{1}{2} v \int \mathrm{d} \mathbf{r} \hat{\rho}_{\mathrm{m}}^{2}\right)
\end{aligned}
$$

where $\int \mathcal{D} g$ denotes the functional integral over the function $g(\mathbf{r})$. The first term in the exponent is the Wiener measure representing the connectivity of the polymer random walk 49], where $\dot{\mathbf{r}}_{l}(s)$ is the derivative of $\mathbf{r}_{l}(s)$ with respect to the monomer index $s$ and the Kuhn length $a$ is the effective monomer length. In writing this term, we have assumed that the charged chains can be modeled as flexible chains. This assumption can be more easily justified for semi-dilute solutions of weakly charged chains.

The second term is the electrostatic interaction term where $\beta=1 / k_{B} T$ is the inverse thermal energy, $v_{\mathrm{c}}(\mathbf{r})=1 / \varepsilon|\mathbf{r}|$ is the Coulomb interaction, $\varepsilon$ is the dielectric constant of the solution and $\hat{\rho}_{\mathrm{c}}(\mathbf{r})$ is the local charge density operator including all charges in the solution (charged monomers, co-ions and counter-ions):

$$
\hat{\rho}_{\mathrm{c}}(\mathbf{r})=\sum_{l=1}^{M} \int_{0}^{N} \mathrm{~d} s q_{l}(s) \delta\left(\mathbf{r}-\mathbf{r}_{l}(s)\right)+\sum_{i=1}^{N^{+}} e \delta\left(\mathbf{r}-\mathbf{R}_{i}^{+}\right)-\sum_{j=1}^{N^{-}} e \delta\left(\mathbf{r}-\mathbf{R}_{j}^{-}\right)
$$

where $q_{l}(s)$ is the random variable denoting the charge carried by the $s$ monomer along the $l$ chain. In the next section we will consider several charge distributions for $q_{l}(s)$. For example, in the smeared model, $q_{l}(s)=p e$ is a constant independent of the position $s$ along the chain and the chain index $l$.

The last term in eq. 1 is the excluded volume repulsion between monomers, where $v \sim a^{3}$ is the excluded volume parameter and

$$
\hat{\rho}_{\mathrm{m}}(\mathbf{r})=\sum_{l=1}^{M} \int_{0}^{N} \mathrm{~d} s \delta\left(\mathbf{r}-\mathbf{r}_{l}(s)\right)
$$

is the local monomer concentration operator.

It is possible to integrate out the microscopic degrees of freedom in the partition function eq. 1 1 by introducing two

pairs of collective coordinates: (i) the local monomer concentration $\rho_{\mathrm{m}}(\mathbf{r})$ and its conjugate field $\varphi_{\mathrm{m}}(\mathbf{r})$; and, (ii) the local charge density $\rho_{\mathrm{c}}(\mathbf{r})$ and its conjugate field $\varphi_{\mathrm{c}}(\mathbf{r})$. This is done using the following identity 


$$
1=\int \mathcal{D} \rho_{\mathrm{m}} \delta\left(\rho_{\mathrm{m}}-\hat{\rho}_{\mathrm{m}}\right)=\int \mathcal{D} \rho_{\mathrm{m}} \mathcal{D} \varphi_{\mathrm{m}} \exp \left(i v \int \mathrm{d} \mathbf{r} \rho_{\mathrm{m}} \varphi_{\mathrm{m}}-i v \sum_{l=1}^{M} \int_{0}^{N} \mathrm{~d} s \varphi_{\mathrm{m}}\left[\mathbf{r}_{l}(s)\right]\right)
$$

for $\rho_{\mathrm{m}}(\mathbf{r})$ and an analogous identity for $\rho_{\mathrm{c}}(\mathbf{r})$. We will see below that $i \varphi_{\mathrm{c}}(\mathbf{r})$ is in fact the electrostatic potential. The functional integrals over $\rho_{\mathrm{m}}(\mathbf{r})$ and $\rho_{\mathrm{c}}(\mathbf{r})$ are Gaussian integrals and are evaluated exactly, leading to

$$
Z=\int \mathcal{D} \varphi_{\mathrm{m}} \mathcal{D} \varphi_{\mathrm{c}} \exp \left(-\int \mathrm{d} \mathbf{r}\left\{\beta \frac{\varepsilon}{8 \pi}\left|\nabla \varphi_{\mathrm{c}}\right|^{2}+\frac{1}{2} v \varphi_{\mathrm{m}}^{2}\right\}\right) \zeta_{+} \zeta_{-} \zeta_{\mathrm{p}}
$$

where $\zeta_{ \pm}=\left(\int \mathrm{d} \mathbf{r} \mathrm{e}^{\mp i \beta e \varphi_{\mathrm{c}}(\mathbf{r})}\right)^{N^{ \pm}}$and $\zeta_{\mathrm{p}}$ are, respectively, the partition functions of the (small) co-ions, counter-ions and polymer in the presence of the external fields $i \varphi_{\mathrm{c}}(\mathbf{r})$ and $i \varphi_{\mathrm{m}}(\mathbf{r})$. In the thermodynamic limit, where $N^{ \pm}$and the volume $V$ become large while the bulk concentrations $c_{\mathrm{b}}^{ \pm}=N^{ \pm} / V$ remain fixed, $\zeta_{ \pm}$become (up to a normalization constant):

$$
\zeta_{ \pm}=\left[1+\frac{1}{V} \int \mathrm{d} \mathbf{r}\left(\mathrm{e}^{\mp i \beta e \varphi_{\mathrm{c}}(\mathbf{r})}-1\right)\right]^{V c_{\mathrm{b}}^{ \pm}} \longrightarrow \exp \left(c_{\mathrm{b}}^{ \pm} \int \mathrm{d} \mathbf{r}\left\{\mathrm{e}^{\mp i \beta e \varphi_{\mathrm{c}}(\mathbf{r})}-1\right\}\right)
$$

The partition function of the polymer chains $\zeta_{\mathrm{p}}$ in the presence of the two external fields is:

$$
\zeta_{\mathrm{p}}=\int \mathcal{D} \mathbf{r}_{l}(s) \exp \left(-\sum_{l=1}^{M} \int_{0}^{N} \mathrm{~d} s\left\{\frac{3}{2 a^{2}} \dot{\mathbf{r}}_{l}^{2}(s)+i v \varphi_{\mathrm{m}}\left[\mathbf{r}_{l}(s)\right]+i \beta q_{l}(s) \varphi_{\mathrm{c}}\left[\mathbf{r}_{l}(s)\right]\right\}\right)
$$

Note that the calculation of $\zeta_{\mathrm{p}}$ depends on the specific charge distribution for $q_{l}(s)$.

\section{MEAN-FIELD EQUATIONS}

We apply the general formalism introduced above to different monomer charge distributions: smeared, annealed, permuted, quenched and polyampholytes. These charge distributions are applicable to different experimental systems.

\section{A. "Smeared" Polyelectrolytes}

The simplest model of a charge distribution is the so-called smeared polyelectrolyte. For a polyelectrolyte with a fraction $p$ of its monomers being charged, this model assumes that each monomer carries a uniform fractional charge $p e$, where $e$ is the electron charge. Namely, $q_{l}(s)=p e$ for any monomer $s$ on any chain $l$. Without loss of generality we shall assume that the polymer is positively charged.

It is possible here to use a well-known analogy from quantum mechanics to calculate the path integral of eq. 7 . The partition function is analogous to a Feynman integral of the Hamiltonian $\mathcal{H}=-\frac{a^{2}}{6} \nabla^{2}+i v \varphi_{\mathrm{m}}+i \beta p e \varphi_{\mathrm{c}}$ with imaginary time $t \rightarrow i s$. Thus, each eigenstate $\phi_{\nu}$ contributes a term of the form $\exp \left(-M N E_{\nu}\right)$ where $M N$ is the total number of monomers in the solution. In the thermodynamic limit $N \rightarrow \infty$, the ground state dominates over all other eigenstates [50], and $\zeta_{\mathrm{p}}$ reduces to:

$$
\zeta_{\mathrm{p}} \approx \mathrm{e}^{-M N E_{0}}=\exp \left(-\int \mathrm{d} \mathbf{r}\left\{\frac{a^{2}}{6}|\nabla \phi|^{2}+i v \varphi_{\mathrm{m}}(\mathbf{r}) \phi^{2}(\mathbf{r})+i \beta p e \varphi_{\mathrm{c}}(\mathbf{r}) \phi^{2}(\mathbf{r})-\mu_{\mathrm{p}} \phi^{2}(\mathbf{r})\right\}\right)
$$

where $E_{0}$ is the ground-state energy, $\phi(\mathbf{r})$ is the renormalized ground-state eigenfunction and $\mu_{\mathrm{p}}$ is a Lagrange multiplier added in order to ensure the normalization of the wave-function $\phi_{\mathrm{b}}^{2}=\frac{1}{V} \int \mathrm{d} \mathbf{r} \phi^{2}(\mathbf{r}), \phi_{\mathrm{b}}^{2}$ being the bulk monomer concentration. The polymer analog of the wave-function $\phi(\mathbf{r})$ is usually referred to as the polymer order parameter, and the local monomer concentration per unit volume can be shown to be $\rho_{\mathrm{m}}(\mathbf{r})=\phi^{2}(\mathbf{r})$ [2] .

The field $\varphi_{\mathrm{m}}$ can now be integrated out leaving a functional integral only over $\psi \equiv i \varphi_{\mathrm{c}}$, and the partition function reduces to:

$$
Z=\int \mathcal{D} \psi \exp \left(-\beta F_{\mathrm{s}}\right)
$$

where 


$$
\begin{aligned}
\beta F_{\mathrm{s}}=\int \mathrm{d} \mathbf{r}\{ & -\beta \frac{\varepsilon}{8 \pi}|\nabla \psi|^{2}+\sum_{ \pm} c_{\mathrm{b}}^{ \pm}\left(1-\mathrm{e}^{\mp \beta e \psi(\mathbf{r})}\right) \\
& \left.+\frac{a^{2}}{6}|\nabla \phi|^{2}+\frac{1}{2} v \phi^{4}(\mathbf{r})-\mu_{\mathrm{p}} \phi^{2}(\mathbf{r})+\beta p e \psi(\mathbf{r}) \phi^{2}(\mathbf{r})\right\}
\end{aligned}
$$

Note that $\psi(\mathbf{r})$ is identified as the electrostatic potential.

Within a mean-field approximation, the functional integral is dominated by the saddle point given by the condition $\delta F / \delta \psi=0$. This results in a Poisson-Boltzmann (PB) like equation for the electrostatic potential, which includes all charge sources

$$
\nabla^{2} \psi(\mathbf{r})=\frac{8 \pi e}{\varepsilon} c_{\mathrm{b}} \sinh (\beta e \psi)-\frac{4 \pi e}{\varepsilon}\left(p \phi^{2}-p \phi_{\mathrm{b}}^{2} \mathrm{e}^{\beta e \psi}\right)
$$

The right hand side represents the local charge density. The first term is the symmetric 1:1 electrolyte contribution, the second term is associated with the (positive) charges on the polymer chains, while the last term comes form the counter-ions which dissociated from the chains. Note that charge neutrality implies $c_{\mathrm{b}}^{+} \rightarrow c_{\mathrm{b}}$ and $c_{\mathrm{b}}^{-} \rightarrow c_{\mathrm{b}}+p \phi_{\mathrm{b}}^{2}$.

Since $\phi(\mathbf{r})$ is the ground state eigenfunction, it satisfies the variational equation $\delta F / \delta \phi=0$, yielding a self-consistent field (SCF) equation for the polymer order parameter:

$$
\frac{a^{2}}{6} \nabla^{2} \phi(\mathbf{r})=v\left(\phi^{3}-\phi_{\mathrm{b}}^{2} \phi\right)+\beta p e \psi \phi
$$

This is an equation for the density of polymer chains in an external electric potential $\psi$ and with excluded volume interactions characterized by the parameter $v$. In the above equation $\mu_{\mathrm{p}}$ has been substituted in order to have the correct bulk limit: $\psi \rightarrow 0$ and $\phi^{2} \rightarrow \phi_{\mathrm{b}}^{2}$.

\section{B. Annealed Polyelectrolytes}

The derivation presented above for the smeared case can be extended to more realistic annealed charge distributions where charges can dynamically associate and dissociate from the chains. The annealed model describes an experimental system where the monomers have weak acidic (or basic) groups. The $\mathrm{pH}$ of the solution controls the degree of association/dissociation of ions on the polymer chain.

Assuming no charge correlations along the chain, the monomer charge distribution $f\left[q_{l}(s)\right]$ is defined as the nominal probability of the monomer $s$ along the chain $l$ to have a charge $q_{l}(s)$.

$$
f\left[q_{l}(s)\right]=\sum_{j} p_{j} \delta\left(q_{l}(s)-z_{j} e\right)
$$

where the randomly charged chain is described by a set of valencies $\left\{z_{j}\right\}\left(z_{j}=0,1,2, \ldots\right)$ with normalized probabilities $\left\{p_{j}\right\}$ such that $\sum_{j} p_{j}=1$. Here, we concentrate on a simple example of a polyelectrolyte for which each monomer can be either positively charged $(z=1$ and $q=+e)$ with probability $p$ or neutral with probability $1-p$. The charge distribution for each monomer is

$$
f\left[q_{l}(s)\right]=p \delta\left(q_{l}(s)-e\right)+(1-p) \delta\left(q_{l}(s)\right)
$$

It is important to note that for annealed polyelectrolytes, the partition function has to be averaged with respect to the monomer charges since they are in thermal equilibrium with the reservoir. As a result the electrostatic contribution of one monomer in $\zeta_{\mathrm{p}}$ (eq. 面) becomes:

$$
\left\langle\mathrm{e}^{-i \beta q_{l}(s) \varphi_{\mathrm{c}}\left[\mathbf{r}_{l}(s)\right]}\right\rangle_{p}=1-p+p \mathrm{e}^{-i \beta e \varphi_{\mathrm{c}}\left[\mathbf{r}_{l}(s)\right]}
$$

where the average is taken over the charge distribution, $\langle\mathcal{O}\rangle_{p}=\int \mathcal{O}(q) f(q) \mathrm{d} q$, and the annealed free energy becomes

$$
\begin{aligned}
\beta F_{\mathrm{a}}=\int \mathrm{d} \mathbf{r}\{ & -\beta \frac{\varepsilon}{8 \pi}|\nabla \psi|^{2}+\sum_{ \pm} c_{\mathrm{b}}^{ \pm}\left(1-\mathrm{e}^{\mp \beta e \psi(\mathbf{r})}\right) \\
& \left.+\frac{a^{2}}{6}|\nabla \phi|^{2}+\frac{1}{2} v \phi^{4}(\mathbf{r})-\mu_{\mathrm{p}} \phi^{2}(\mathbf{r})-\phi^{2}(\mathbf{r}) \log \left(1-p+p \mathrm{e}^{-\beta e \psi(\mathbf{r})}\right)\right\}
\end{aligned}
$$


The variation of eq. 16 with respect to $\psi(\mathbf{r})$, leads to a PB like equation

$$
\nabla^{2} \psi(\mathbf{r})=\frac{8 \pi e}{\varepsilon} c_{\mathrm{b}} \sinh (\beta e \psi)-\frac{4 \pi e}{\varepsilon}\left(p_{\mathrm{a}} \phi^{2}-p \phi_{\mathrm{b}}^{2} \mathrm{e}^{\beta e \psi}\right)
$$

where

$$
p_{\mathrm{a}}(\mathbf{r}) \equiv p \mathrm{e}^{-\beta e \psi(\mathbf{r})} /\left(1-p+p \mathrm{e}^{-\beta e \psi(\mathbf{r})}\right)
$$

can be interpreted as the annealed charge probability and depends on the local electrostatic potential. Similarly, the variation of eq. 16 with respect to $\phi(\mathbf{r})$ gives a modified SCF equation:

$$
\frac{a^{2}}{6} \nabla^{2} \phi(\mathbf{r})=v\left(\phi^{3}-\phi_{\mathrm{b}}^{2} \phi\right)+\phi \log \left(1-p_{\mathrm{a}}+p_{\mathrm{a}} \mathrm{e}^{\beta e \psi}\right)
$$

Equations 17 and 19 are the annealed equations for the charge distribution eq. 14, similar to eqs. 11 and 12 for the smeared case.

\section{C. "Permuted" Polyelectrolytes}

Another variant of the annealed case is the permuted model for which a fixed number of charges $p N$ are free to move along each chain without dissociating from it. Thus, the total charge on the chain remains constant. This is introduced into the annealed model by adding a constraint in eq. 7 in order to keep the total charge on each of the chain fixed:

$$
\prod_{l} \delta\left(\int_{0}^{N} \mathrm{~d} s q_{l}(s)-N p e\right)=\int \mathrm{d} u_{l} \exp \left(i u_{l} \int_{0}^{N} \mathrm{~d} s\left[q_{l}(s)-p e\right]\right)
$$

where $u_{l}$ is a Lagrange multiplier related to the fixed charge constraint of the chain $l$. Assuming ground state dominance, the polymer partition function becomes:

$$
\begin{aligned}
\zeta_{\mathrm{p}}=\left[\int \mathrm { d } u \operatorname { e x p } \left(-\int \mathrm{d} \mathbf{r}\{\right.\right. & \frac{a^{2}}{6}|\nabla \phi|^{2}+i v \varphi_{\mathrm{m}}(\mathbf{r}) \phi^{2}(\mathbf{r})+i p e u \phi^{2}(\mathbf{r}) \\
& \left.\left.\left.-\mu_{\mathrm{p}} \varphi_{\mathrm{m}}^{2}(\mathbf{r})-\phi^{2}(\mathbf{r}) \log \left(1+\mathrm{e}^{-i p \beta e \varphi_{\mathrm{c}}(\mathbf{r})+i e u}\right)\right\}\right)\right]^{M}
\end{aligned}
$$

where the index $l$ is dropped from the functional integral over $u_{l}$ since the constraint is satisfied separately on each chain. Note that the fraction $p$ of charged monomers is introduced through the constraint (eq. 20). In order to carry out the integration over $u$, we use the identity (similar to eq. đ)

$$
1=\int \mathcal{D} g(\mathbf{r}) \delta(g(\mathbf{r})-u)=\int \mathcal{D} g(\mathbf{r}) \mathcal{D} h(\mathbf{r}) \exp \left(i \int \mathrm{d} \mathbf{r} h(\mathbf{r}) g(\mathbf{r})-i \int \mathrm{d} \mathbf{r} h(\mathbf{r}) u\right)
$$

A sequence of saddle point approximations for $g(\mathbf{r}), h(\mathbf{r})$ and $u$ leads to the following mean-field free energy for the permuted case:

$$
\beta F_{\mathrm{p}}=\beta F_{\mathrm{s}}+\int \mathrm{d} \mathbf{r}\{p \log p+(1-p) \log (1-p)\} \phi^{2}(\mathbf{r})
$$

This correction term represents the translational entropy of the charges on the chain [23]. However, the last term being quadratic in $\phi$ and independent of $\psi$, it only shifts the chemical potential $\mu_{p}$ without affecting the differential equations 11 and 12 . 


\section{Quenched Polyelectrolytes}

In the quenched model the charge distribution is frozen. Experimentally, this corresponds to heterogeneous copolymers with a random sequence of charged and neutral monomers. The specific sequence of each copolymer is determined during the polymerization stage and represents one possible realization of the random distribution. Various physical quantities are then calculated by averaging over this random distribution. Instead of averaging the partition function, one should average the free energy itself over the random charge distribution. A standard method in quenched systems is the replica trick [52] based on the following identity:

$$
\langle\log Z\rangle=\lim _{n \rightarrow 0} \frac{\mathrm{d}\left\langle Z^{n}\right\rangle}{\mathrm{d} n}
$$

where $\langle\ldots\rangle$ indicates an average over the quenched disorder and the $n$-th power of the partition function introduces $n$ replicas of the system all having the same monomer charge distribution. Generalizing the above approach we obtain:

$$
\left\langle Z^{n}\right\rangle=\int \mathcal{D} \varphi_{\mathrm{m}}^{\alpha} \mathcal{D} \varphi_{\mathrm{c}}^{\alpha} \exp \left(-\sum_{\alpha=1}^{n} \int \mathrm{d} \mathbf{r}\left\{\beta \frac{\varepsilon}{8 \pi}\left|\nabla \varphi_{\mathrm{c}}^{\alpha}\right|^{2}+\sum_{ \pm} c_{\mathrm{b}}^{ \pm}\left(1-\mathrm{e}^{\mp i \beta e \varphi_{\mathrm{c}}^{\alpha}}\right)+\frac{1}{2} v\left(\varphi_{\mathrm{m}}^{\alpha}\right)^{2}\right\}\right) \times\left\langle\zeta_{n}\right\rangle
$$

where

$$
\begin{aligned}
\left\langle\zeta_{n}\right\rangle & =\int \mathcal{D} \mathbf{r}_{l}^{\alpha}(s) \exp \left(-\sum_{\alpha=1}^{n} \sum_{l=1}^{M} \int_{0}^{N} \mathrm{~d} s\left\{\frac{3}{2 a^{2}}\left(\dot{\mathbf{r}}_{l}^{\alpha}(s)\right)^{2}+i v \varphi_{\mathrm{m}}^{\alpha}\left[\mathbf{r}_{l}^{\alpha}(s)\right]\right\}\right) \\
& \times \exp \left(\sum_{l=1}^{M} \int_{0}^{N} \mathrm{~d} s \log \left(1-p+p \mathrm{e}^{-i \beta e \sum_{\alpha} \varphi_{\mathrm{c}}^{\alpha}\left[\mathbf{r}_{l}^{\alpha}(s)\right]}\right)\right)
\end{aligned}
$$

and the superscript $\alpha=1, \ldots, n$ is the label of the replica $\alpha$. A mean-field estimate of $\zeta_{n}$ can be obtained by using again the quantum-mechanical analogy. An additional complication is that the effective Hamiltonian here is a many-body one as it couples different replicas:

$$
\mathcal{H}_{n}=\sum_{\alpha=1}^{n}\left\{-\frac{a^{2}}{6} \nabla_{\alpha}^{2}+i v \varphi_{\mathrm{m}}^{\alpha}\right\}-\log \left(1-p+p \mathrm{e}^{-i \beta e \sum_{\alpha} \varphi_{\mathrm{c}}^{\alpha}}\right)
$$

Assuming no replica symmetry breaking, we use a Hartree approximation to express the ground state of the many-body eigenfunction $\phi_{n}\left(\left\{\mathbf{r}^{\alpha}\right\}\right)=\prod_{\alpha=1}^{n} \phi\left(\mathbf{r}^{\alpha}\right)$, in terms of single-body eigenfunctions $\phi\left(\mathbf{r}^{\alpha}\right)$ :

$$
\begin{aligned}
\zeta_{n} \approx & \mathrm{e}^{-M E_{n}}=\exp \left(-n M \int \mathrm{d} \mathbf{r}\left\{\frac{a^{2}}{6}|\nabla \phi|^{2}+i v \varphi_{\mathrm{m}} \phi^{2}-\mu_{\mathrm{p}} \varphi_{\mathrm{m}}^{2}\right\}\right) \\
& \times \exp \left(M \int \mathrm{d} \mathbf{r}^{\alpha} \prod_{\alpha=1}^{n} \phi^{2}\left(\mathbf{r}^{\alpha}\right) \log \left(1-p+p \mathrm{e}^{-i \beta e \sum_{\alpha} \varphi_{\mathrm{c}}\left(\mathbf{r}^{\alpha}\right)}\right)\right)
\end{aligned}
$$

The path integrals over $\varphi_{\mathrm{m}}^{\alpha}(\mathbf{r})$ can be evaluated exactly, whereas the path integrals over $\varphi_{\mathrm{c}}^{\alpha}(\mathbf{r})$ can be approximated by their saddle point values. Since we assumed no replica symmetry breaking, all saddle point functions are identical: $i \varphi_{\mathrm{c}}^{\alpha}(\mathbf{r}) \equiv \psi(\mathbf{r})$. When we take into account the coupling with the polyelectrolyte reservoir, this equation reduces to the annealed PB equation (eq. 17). Similarly, the SCF equation reduces to eq. 19 .

\section{E. Annealed Polyampholytes}

Finally, the mean-field formalism can be generalized in a straightforward way to treat polyampholytes, consisting of negatively and positively charged monomers. A general polyampholyte is described by a set of valencies $\left\{z_{j}\right\}$ $\left(z_{j}=0, \pm 1, \pm 2, \ldots\right)$ with probabilities $\left\{p_{j}\right\}$, where $\sum_{j} p_{j}=1$. The statistical distribution of the charges can be either annealed, quenched or permuted. For simplicity, we consider only the annealed case where the contribution of the charged monomers to the free energy becomes

$$
\beta F_{\mathrm{pa}}^{e l}=-\int \mathrm{d} \mathbf{r} \phi^{2}(\mathbf{r}) \log \left(\sum_{j} p_{j} \mathrm{e}^{-\beta z_{j} e \psi(\mathbf{r})}\right)
$$


and should be compared to the last term in eq. 16. The modified PB equation (eq. 17) is now:

$$
\nabla^{2} \psi(\mathbf{r})=\frac{8 \pi e}{\varepsilon} c_{\mathrm{b}} \sinh (\beta e \psi)-\frac{4 \pi e}{\varepsilon}\left(z_{\mathrm{a}} \phi^{2}-\bar{z} \phi_{\mathrm{b}}^{2} \mathrm{e}^{\beta e \psi}\right)
$$

where $\bar{z} \equiv \sum_{j} p_{j} z_{j}$ is the average monomer charge (in units of $e$ ) and

$$
z_{\mathrm{a}}(\mathbf{r})=\frac{\sum_{j} p_{j} z_{j} \mathrm{e}^{-\beta z_{j} e \psi(\mathbf{r})}}{\sum_{j} p_{j} \mathrm{e}^{-\beta z_{j} e \psi(\mathbf{r})}}
$$

is the annealed (weighted) monomer valence, which is nothing but the Boltzmann average of the monomer charge distribution. Finally, the SCF equation (eq. 19) is:

$$
\frac{a^{2}}{6} \nabla^{2} \phi(\mathbf{r})=v\left(\phi^{3}-\phi_{\mathrm{b}}^{2} \phi\right)-\phi \log \left(\sum_{j} p_{j} \mathrm{e}^{-\beta z_{j} e \psi}\right)
$$

\section{DISCUSSION OF THE CHARGE MODELS}

In the previous section we derived the free energies and mean-field equations for different charge distributions, by path integral methods.

The smeared case: The smeared free energy can be separated into uncharged polymer and Coulombic contributions 455, $F_{\mathrm{s}}=F_{\mathrm{pol}}+F_{\mathrm{el}}$. The polymer part is:

$$
F_{\mathrm{pol}}=k_{B} T \int \mathrm{d} \mathbf{r}\left\{\frac{a^{2}}{6}|\nabla \phi|^{2}+\frac{1}{2} v \phi^{4}(\mathbf{r})-\mu_{\mathrm{p}} \phi^{2}(\mathbf{r})\right\}
$$

This is the Edwards free energy for polymer solutions expressed in terms of the polymer order parameter $\phi(\mathbf{r})$, which is the square root of the monomer concentration, $\rho_{m}=\phi^{2}$. The first term represents the stiffness of the polymer chains, the second takes into account the excluded volume in good solvent conditions, while the last term represents the coupling to a reservoir of monomers with chemical potential $\mu_{\mathrm{p}}$.

The Coulombic free energy reads:

$$
F_{\mathrm{el}}=\int \mathrm{d} \mathbf{r}\left\{-\frac{\varepsilon}{8 \pi}|\nabla \psi|^{2}+k_{B} T \sum_{ \pm} c_{\mathrm{b}}^{ \pm}\left(1-\mathrm{e}^{\mp \beta e \psi(\mathbf{r})}\right)+p e \psi(\mathbf{r}) \phi^{2}(\mathbf{r})\right\}
$$

This free energy contains the electrostatic interactions between all charges (small ions and charged monomers), as well as the translational entropy of the small ions in solution. In the above expression the independent fields are the electric potential $\psi$ and polymer order parameter $\phi$. The small ion concentrations are uniquely determined by the electric potential through the Boltzmann weight: $c^{ \pm}(\mathbf{r})=c_{\mathrm{b}}^{ \pm} \exp (\mp \beta e \psi(\mathbf{r}))$.

The same free energy $F_{\text {el }}$ can be obtained from a more direct approach as will be shown for the smeared case. It is convenient to express the free energy in terms of the total charge density: $\rho_{c}=e c^{+}-e c^{-}+p e \phi^{2}$

$$
\begin{aligned}
\tilde{F}_{\mathrm{el}} & =\frac{1}{2} \int \mathrm{d} \mathbf{r} \mathrm{d} \mathbf{r}^{\prime} \frac{\rho_{c}(\mathbf{r}) \rho_{c}\left(\mathbf{r}^{\prime}\right)}{\varepsilon\left|\mathbf{r}-\mathbf{r}^{\prime}\right|} \\
& +k_{B} T \int \mathrm{d} \mathbf{r}\left\{c^{+}\left(\log c^{+}-1\right)+c^{-}\left(\log c^{-}-1\right)\right\}-\int \mathrm{d} \mathbf{r}\left\{\mu_{+} c^{+}+\mu_{-} c^{-}\right\}
\end{aligned}
$$

The free energy $F_{\text {el }}$ of eq. 34 should be distinguished from $\tilde{F}_{\text {el }}$. The former depends on $\psi$ and $\phi$ while the latter depends on $c^{ \pm}$and $\phi$. For the latter, the electric potential can be defined as

$$
\psi(\mathbf{r})=\int \mathrm{d} \mathbf{r}^{\prime} \frac{\rho_{c}\left(\mathbf{r}^{\prime}\right)}{\varepsilon\left|\mathbf{r}-\mathbf{r}^{\prime}\right|}
$$

and it satisfies the Poisson equation 


$$
\nabla^{2} \psi=-\frac{4 \pi}{\varepsilon} \rho_{c}
$$

Minimizing $\tilde{F}_{\text {el }}$ with respect to $c^{ \pm}$we obtain the equilibrium charge distribution of the small ions:

$$
c^{ \pm}(\mathbf{r})=c_{\mathrm{b}}^{ \pm} \exp (\mp \beta e \psi(\mathbf{r})
$$

where $\mu_{ \pm}=k_{B} T \log c_{\mathrm{b}}^{ \pm}$.

Substituting the above equilibrium condition back into eq. 35 we obtain

$$
\begin{gathered}
\tilde{F}_{\mathrm{el}}=\frac{1}{2} \int \mathrm{d} \mathbf{r} p e \phi^{2} \psi-\frac{1}{2} \int \mathrm{d} \mathbf{r}\left(e c^{+}-e c^{-}\right) \psi \\
-k_{B} T \int \mathrm{d} \mathbf{r}\left\{c_{\mathrm{b}}^{+}\left(\mathrm{e}^{-\beta e \psi}-1\right)+c_{\mathrm{b}}^{-}\left(\mathrm{e}^{\beta e \psi}-1\right)\right\}
\end{gathered}
$$

From the Poisson equation 37 we can express $c^{+}-c^{-}$in terms of $\nabla^{2} \psi$ and $\phi$. Integration by parts of the term $\psi \nabla^{2} \psi$ yields exactly the first term of eq. 34 with the correct negative sign 455.51.

The annealed case: The second type of charge distribution is the annealed one where each monomer can be either charged or neutral with bare probabilities $p$ and $1-p$, respectively. Its free energy eq. 16 is similar to the smeared one except for the coupling term between the charged monomers and the local electric potential. The difference can be understood in the following way: in the annealed case different charge configurations will contribute to the free energy and one needs to trace over those configurations in the partition function before the free energy is calculated. In the smeared case there is only one charge configuration where every monomer is assigned a fractional charge pe, whereas in the annealed case $p$ represents the bare probability of dissociation and $p_{a}=p \exp (-\beta e \psi) /(1-p+p \exp (-\beta e \psi))$ is the effective probability as can be seen in eqs. 17 and 19 .

In experiments the effective probability $p_{a}$ is related to the $\mathrm{pH}$ of the solution via

$$
\mathrm{pH}=\mathrm{pK}_{0}+\log _{10} \frac{p_{a}}{1-p_{a}}
$$

where $\mathrm{pK}_{0}=-\log _{10} \mathrm{~K}_{0}$ and $K_{0}$ is the dissociation constant. For example, in the case of weak alkaline monomers

$$
\mathrm{AOH} \rightleftharpoons \mathrm{A}^{+}+\mathrm{OH}^{-}
$$

$\mathrm{K}_{0}$ is given by

$$
\mathrm{K}_{0}=\frac{\left[\mathrm{A}^{+}\right]\left[\mathrm{OH}^{-}\right]}{[\mathrm{AOH}]}
$$

From eq. 40 one can easily obtain $p_{a}$ as a function of the $\mathrm{pH}$.

At low electrostatic potentials $|\beta e \psi| \ll 1$, the annealed free energy can be expanded in powers of $\psi$. The first term is equal to the smeared free energy $F_{\mathrm{s}}$, while the next term is always negative

$$
\beta F_{\mathrm{a}} \simeq \beta F_{\mathrm{s}}-\frac{1}{2} p(1-p) \beta^{2} e^{2} \int \mathrm{d} \mathbf{r} \psi^{2}(\mathbf{r}) \phi^{2}(\mathbf{r})<\beta F_{\mathrm{s}}
$$

The fact that $F_{\mathrm{a}}<F_{\mathrm{s}}$ is related to the convexity of the free energy. Indeed, the annealed case has more degrees of freedom and allows a better minimization.

The permuted case: The permuted model is a variant of the annealed case. It models either mobile charges which can hop along the chain or charges which associate and dissociate while keeping the total amount of charge fixed on each chain.

The free energy for the permuted case can be written as the smeared free energy eq. 10 plus an additional term of entropic origin:

$$
F_{\mathrm{p}}=F_{\mathrm{s}}+k_{B} T \int \mathrm{d} \mathbf{r}\{p \log p+(1-p) \log (1-p)\} \phi^{2}(\mathbf{r})
$$

The correction is due to the translational entropy of the charges along the chains. It amounts to a shift in the polymer chemical potential and thus does not affect the mean field equation. 
The correspondence between the permuted and the smeared models was not emphasized in previous works. It can be interpreted as a tendency of the charges in the permuted model to spread uniformly along the linear chain. However, as was discussed earlier [23, changing the pH of the solution (e.g., by titration) can lead to non trivial dependence of $\mu_{p}$ on the physical parameters, since the reservoir concentration changes in a titration process and will affect $\mu_{\mathrm{p}}$.

The quenched case: In order to obtain the equilibrium state of chains with frozen (quenched) charge distributions, the free energy has to be averaged over all possible charge configurations. As was shown in the previous section, annealed and quenched polyelectrolytes in contact with an infinite reservoir of chains have the same mean field free energy.

The physical meaning of this result can be explained in the following way [53: when quenched polymers are allowed to exchange with a bulk reservoir, containing all possible configurations, the system picks up the optimal configurations from the bulk. When the polymers are not coupled to an infinite reservoir the annealed and quenched cases are different. Note that the dynamics of annealed and quenched polyelectrolytes can differ considerably, but this is beyond the scope of the current work.

The annealed polyampholyte case: This situation corresponds to monomers which can carry a positive or negative charges of valency $z_{j}=0, \pm 1, \pm 2, \ldots$ with probability $p_{j}$. As was derived in the previous section the electrostatic part of the free energy is given by:

$$
F_{\mathrm{pa}}^{e l}=-k_{B} T \int \mathrm{d} \mathbf{r} \phi^{2}(\mathbf{r}) \log \left(\sum_{j} p_{j} \mathrm{e}^{-\beta z_{j} e \psi(\mathbf{r})}\right)
$$

For low electrostatic potentials, an expansion of the above polyampholyte free energy yields:

$$
F_{\text {pa }}^{e l} \simeq \bar{z} e \int \mathrm{d} \mathbf{r} \psi(\mathbf{r}) \phi^{2}(\mathbf{r})-\frac{1}{2} \beta \sigma^{2} e^{2} \int \mathrm{d} \mathbf{r} \psi^{2}(\mathbf{r}) \phi^{2}(\mathbf{r})
$$

The first term is linear in $\psi$ and reduces to the smeared contribution where $\bar{z} e$ replaces pe as the average monomer charge. The second term is a negative correction (as expected) which depends on the statistical variance of the charge distribution

$$
\sigma^{2}=\sum_{j} p_{j} z_{j}^{2}-\left(\sum_{j} p_{j} z_{j}\right)^{2}
$$

This term affects the monomer-monomer correlations and $S(q)$ as discussed in the following section.

The general distribution treated here has a few simple and useful limits:

1. The smeared model (Sec. III.A) is exactly recovered for $p_{1}=1$ and $z_{1}=p$ yielding $\bar{z}=p$ and $\sigma^{2}=0$.

2. The annealed model (Sec. III.B) is obtained for $p_{1}=p, z_{1}=1$ and $p_{2}=1-p, z_{2}=0$; $\bar{z}$ being simply $p, z_{\mathrm{a}}$ being $p_{\mathrm{a}}$, and the variance $\sigma^{2}=p(1-p)$.

3. A trimodal charge distribution can be a good representation for some polyampholyte systems.

$$
f(q)=p_{+} \delta(q-e)+p_{0} \delta(q)+p_{-} \delta(q+e)
$$

where $p_{0}=1-p_{+}-p_{-}$. Each monomer can be either positively charged, negatively charged or neutral with probabilities $p_{+}, p_{-}$and $p_{0}$, respectively. The trimodal distribution is characterized by two independent parameters. These can be the first two moments: the average $\bar{z}=p_{+}-p_{-}$and the variance ("width") $\sigma^{2}=p_{+}\left(1-p_{+}\right)+p_{-}\left(1-p_{-}\right)+2 p_{+} p_{-}$. Increasing the weight of $p_{0}$ means that the polyampholyte becomes weakly charged, while increasing the weight of $p_{+}$(or $p_{-}$) means that the polyampholyte becomes more asymmetric and resembles more a true polyelectrolyte.

4. A bimodal distribution of polyampholytes where each monomer carries either a $+e$ charge or $-e$ one, with probabilities $p$ and $1-p$, respectively.

$$
f(q)=p \delta(q-e)+(1-p) \delta(q+e)
$$

with $\bar{z}=2 p-1$ and $\sigma^{2}=4 p(1-p)$. Note that for this fully charged polyampholyte the same parameter $p$ characterizes both the average $\bar{z}$ and the variance $\sigma^{2}$, so that $\sigma^{2}=1-\bar{z}^{2}$. The distribution is symmetric around $p=1 / 2$. As $|p-1 / 2|$ increases, $|\bar{z}|$ increases, $\sigma^{2}$ decreases, and the polyampholyte resembles more and more a polyelectrolyte with a net charge. 
It is of interest to consider explicitly the symmetric bimodal case mentioned above having no net charge $p=1 / 2$, $\bar{z}=0$ and $\sigma^{2}=1$ is maximal. The $\mathrm{PB}$ equation (eq. 30) can be written as

$$
\nabla^{2} \psi(\mathbf{r})=\frac{8 \pi e}{\varepsilon} c_{\mathrm{b}} \sinh (\beta e \psi)+\frac{4 \pi e}{\varepsilon} \phi^{2} \tanh (\beta e \psi)
$$

and the SCF equation (eq. 32) is:

$$
\frac{a^{2}}{6} \nabla^{2} \phi(\mathbf{r})=v\left(\phi^{3}-\phi_{\mathrm{b}}^{2} \phi\right)-\phi \log [\cosh (\beta e \psi)]
$$

The second term in eq. 50 represents the contribution of the charged monomers to the local charge density. At low potentials $|\beta e \psi| \ll 1$ the polymer charge density is $e \phi^{2} \tanh (\beta e \psi) \simeq e \phi^{2} \sinh (\beta e \psi)$. Comparing, in this limit, the two terms on the right hand side of eq. 50, the polyampholyte can be viewed as a symmetric electrolyte [8] whose bulk concentration is not a constant but determined by the local monomer concentration. For a monovalent electrolyte, the local concentrations of positive and negative ions obey a Boltzmann distribution $c^{ \pm}(\mathbf{r})=c_{\mathrm{b}} \exp (\mp \beta e \psi)$, where the equilibrium distribution is achieved by exchange of ions with the reservoir. For the annealed polyampholytes, the concentrations of positive and negative monomers $\rho_{\mathrm{m}}^{ \pm}(\mathbf{r})$ behave in a similar way (for weak potentials), $\rho_{\mathrm{m}}^{ \pm}(\mathbf{r}) \simeq$ $\frac{1}{2} \phi^{2} \exp (\mp \beta e \psi)$, but the mechanism is different. For the latter, the equilibrium distribution is achieved by ionizing the monomers, and this process is limited by the monomer concentration, whereas for the former case, the reservoir contains an infinite amount of ions. This difference becomes evident at high potentials where $\tanh (\beta e \psi)$ saturates to +1 or -1 , depending on the sign of the potential. Under these extreme conditions the polyampholytes are no longer neutral. Instead, they are fully ionized.

\section{STRUCTURE FACTOR WITHIN RPA}

Density-density correlations are measured in scattering experiments [27,28] and can be calculated using the random phase approximation (RPA) 21 26]. This is done by considering small fluctuations of the homogeneous bulk state. Since we are interested in the monomer-monomer density correlations, we do not perform the integration over $\rho_{\mathrm{m}}(\mathbf{r})$

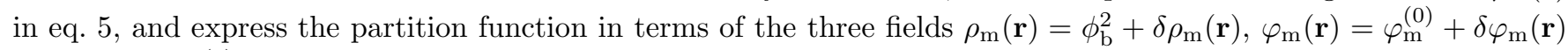
and $\varphi_{\mathrm{c}}(\mathbf{r})=\varphi_{\mathrm{c}}^{(0)}+\delta \varphi_{\mathrm{c}}(\mathbf{r})$. As introduced in Sec. II, $\varphi_{\mathrm{m}}$ is the conjugate field of the local monomer concentration $\rho_{\mathrm{m}}$ and $\varphi_{\mathrm{c}}$ is the electric potential conjugate to the charge density $\rho_{\mathrm{c}}$. Note that $\varphi_{\mathrm{c}}^{(0)}=0$ in the bulk.

The free energy is then expanded to second order in these fluctuations. Since the linear terms in $\delta \rho_{\mathrm{m}}, \delta \varphi_{\mathrm{m}}$ and $\delta \varphi_{\mathrm{c}}$ cancel out, the first non-zero corrections are of second order.

It is more convenient to write this expansion in Fourier space:

$$
\delta \rho_{\mathrm{m}}(\mathbf{r})=\int \frac{\mathrm{d} \mathbf{q}}{(2 \pi)^{3}} \delta \rho_{\mathrm{m}}(\mathbf{q}) \mathrm{e}^{i \mathbf{q} \cdot \mathbf{r}}
$$

and similarly for $\delta \varphi_{\mathrm{m}}$ and $\delta \varphi_{\mathrm{c}}$.

\section{A. Smeared $S(q)$}

Using the smeared free energy, the expansion yields

$$
\begin{aligned}
\beta F_{\mathrm{s}}\left(\delta \rho_{\mathrm{m}}, \delta \varphi_{\mathrm{m}}, \delta \varphi_{\mathrm{c}}\right) \simeq & \int \frac{\mathrm{d} \mathbf{q}}{(2 \pi)^{3}}\left\{v \delta \rho_{\mathrm{m}}(-\mathbf{q}) \delta \rho_{\mathrm{m}}(\mathbf{q})+v^{2} \phi_{\mathrm{b}}^{2} S_{0}(q) \delta \varphi_{\mathrm{m}}(-\mathbf{q}) \delta \varphi_{\mathrm{m}}(\mathbf{q})\right. \\
& +\beta \frac{\varepsilon}{4 \pi}\left[q^{2}+\kappa_{s}^{2}+p \kappa_{p}^{2} S_{0}(q)\right] \delta \varphi_{\mathrm{c}}(-\mathbf{q}) \delta \varphi_{\mathrm{c}}(\mathbf{q}) \\
& \left.+\beta \operatorname{pev} \phi_{\mathrm{b}}^{2} S_{0}(q) \delta \varphi_{\mathrm{m}}(-\mathbf{q}) \delta \varphi_{\mathrm{c}}(\mathbf{q})-i v \delta \rho_{\mathrm{m}}(-\mathbf{q}) \delta \varphi_{\mathrm{m}}(\mathbf{q})\right\}
\end{aligned}
$$

where the integral is over the wavevector $\mathbf{q}$, and $S_{0}(q)$ is the structure factor of Gaussian chains. For chains of length $N$, it is equal to $S_{0}(q)=N D\left(\frac{1}{6} a^{2} q^{2} N\right)$ where $D(\zeta)=\left(2 / \zeta^{2}\right)\left(\mathrm{e}^{-\zeta}+\zeta-1\right)$ is the Debye function [2]. For infinitely long chains $N \rightarrow \infty$, the structure factor is independent of the chain length, $S_{0}^{-1}(q)=\frac{1}{12} a^{2} q^{2}$. 
The Debye-Hückel screening length has two contributions:

$$
\kappa_{\mathrm{tot}}^{2}=\kappa_{s}^{2}+p \kappa_{p}^{2}=8 \pi l_{B} c_{\mathrm{b}}+4 \pi l_{B} p \phi_{\mathrm{b}}^{2}
$$

the first contribution arises from the 1:1 symmetric electrolyte while the second one comes from the polymer counterions, $l_{B}=e^{2} / \varepsilon k_{B} T$ being the Bjerrum length, equal to about $7 \AA$ at room temperature.

The Fourier transform of the monomer-monomer correlations $\left\langle\delta \rho_{\mathrm{m}}(\mathbf{r}) \delta \rho_{\mathrm{m}}(\mathbf{0})\right\rangle$ is proportional to the experimentally measured structure factor $S(q)$. It can now be calculated as a Gaussian integral giving:

$$
S_{\mathrm{s}}^{-1}(q)=\frac{\phi_{\mathrm{b}}^{2}}{\left\langle\delta \rho_{\mathrm{m}}(-\mathbf{q}) \delta \rho_{\mathrm{m}}(\mathbf{q})\right\rangle}=S_{0}^{-1}(q)+v \phi_{\mathrm{b}}^{2}+\frac{p^{2} \kappa_{p}^{2}}{q^{2}+\kappa_{s}^{2}+p \kappa_{p}^{2}}
$$

Similar expressions were obtained in previous works [21 25], using somewhat different derivations.

\section{B. Annealed $S(q)$ for Polyelectrolytes and Polyampholytes}

Repeating the above calculation for the annealed case leads to the same form for $S(q)$ where $\kappa_{\text {tot }}^{2}$ is replaced by $\kappa_{\text {tot }}^{2}+p(1-p) \kappa_{p}^{2}$ in eq. 55 .

$$
S_{\mathrm{a}}^{-1}(q)=S_{0}^{-1}(q)+v \phi_{\mathrm{b}}^{2}+\frac{p^{2} \kappa_{p}^{2}}{q^{2}+\kappa_{s}^{2}+p(2-p) \kappa_{p}^{2}}
$$

The enhanced screening here is due to the additional annealed degrees of freedom of the charges on the polymer chains. Local fluctuations of the monomer charge density effectively increase the local ion concentration leading to stronger screening.

The structure factor is also calculated for annealed polyampholytes. It amounts to replacing $\kappa_{\text {tot }}^{2}$ by $\kappa_{s}^{2}+\left(|\bar{z}|+\sigma^{2}\right) \kappa_{p}^{2}$ and $p^{2} \kappa_{p}^{2}$ by $\bar{z}^{2} \kappa_{p}^{2}$ in eq. 55 leading to:

$$
S_{\mathrm{pa}}^{-1}(q)=S_{0}^{-1}(q)+v \phi_{\mathrm{b}}^{2}+\frac{\bar{z}^{2} \kappa_{p}^{2}}{q^{2}+\kappa_{s}^{2}+\left(|\bar{z}|+\sigma^{2}\right) \kappa_{p}^{2}}
$$

For neutral (symmetric) polyampholytes, $\bar{z}=0$, expression 57 is the same as $S^{-1}(q)$ of neutral polymers. We note that this is an outcome of the RPA which neglects higher order charge correlations. However, the mean field equations themselves as well as the free energy 45 depend on the charge distribution and, in particular, on the variance $\sigma^{2}$.

At high charge fraction $|\bar{z}|$ and low salt concentration, the structure factor $S(q)$ exhibits a peak at a finite wavenumber $q_{0}>0$ satisfying

$$
\left[q_{0}^{2}+\kappa_{s}^{2}+\left(|\bar{z}|+\sigma^{2}\right) \kappa_{p}^{2}\right]^{2}=12 \bar{z}^{2} \kappa_{p}^{2} / a^{2}
$$

This peak is characteristic of polyelectrolyte solutions at low salt concentration. Since $S^{-1}(q)$ is the energy of the $q$ mode density fluctuations, a maximum in $S(q)$ corresponds to the lowest energy fluctuation. Here $q_{0}>0$ results from the competition between the first term in 57, originating from the polymer elasticity, and the last term due to the electrostatic interactions screened by the small ions. For $q$ values $\kappa<q<a^{-1}$, the peak $q_{0}$ can be observed. For example, in the absence of salt, the condition for having a peak at $q_{0}>0$ is

$$
0<\frac{\sigma^{2}}{|\bar{z}|}<\frac{2 \sqrt{3}}{\kappa_{p} a}-1
$$

The upper bound is bigger than zero as long as $\kappa_{p}^{-1} \geq a$ recalling that $\kappa_{p}^{-1}=1 / \sqrt{4 \pi l_{B} \phi_{\mathrm{b}}^{2}}$ and $a$ is the monomer size. The above inequality is satisfied for highly asymmetric polyampholytes, resembling polyelectrolytes (see case 3 of the preceding section). In the opposite limit, low $|\bar{z}|$ and/or high $\sigma$, the random polyampholyte behaves essentially as a neutral polymer $\left(q_{0} \rightarrow 0\right)$. For fully charged polyampholytes (case 4 of the preceding section), $\sigma^{2} /|\bar{z}|=(1-$ $\left.\bar{z}^{2}\right) /|\bar{z}|$ yielding the same conclusion. As long as the net charge $|\bar{z}|$ is large enough, the polyampholytes resemble polyelectrolytes and $S(q)$ will exhibit a peak at finite $q_{0}$.

In Fig. 2 the structure factor $S(q)$ is plotted as function of the wavenumber $q$ for smeared polyelectrolytes $\left(\sigma^{2}=0\right)$ for different salt concentrations and charge fractions $\bar{z}$. The structure factor at wavenumber $q=0$ is proportional to the osmotic compressibility. As depicted in Fig. 2, $S(q=0)$ increases upon addition of salt, while the peak position at $q_{0}$ shifts to smaller wavenumbers until the peak disappears and $S(q)$ becomes a monotonous decreasing function. The inset of Fig. 2 shows the effect of decreasing the average monomer charge $|\bar{z}|$. The peak increases and shifts towards smaller values of $q$. 


\section{Mesophases in Bad Solvent}

We end this section by examining polyelectrolytes and polyampholytes in bad solvent conditions. The excluded volume parameter $v$ is negative leading to collapse (and segregation) of the chains, and higher order virial terms have to be included in the free energy. Assuming that the third order virial coefficient $w$ is positive, with a contribution of $\frac{1}{6} w \rho_{\mathrm{m}}^{3}$ to the free energy, we obtain

$$
S_{\mathrm{pa}}^{-1}(q)=S_{0}^{-1}(q)-|v| \phi_{\mathrm{b}}^{2}+w \phi_{\mathrm{b}}^{4}+\frac{\bar{z}^{2} \kappa_{p}^{2}}{q^{2}+\kappa_{s}^{2}+\left(|\bar{z}|+\sigma^{2}\right) \kappa_{p}^{2}}
$$

An analogous expression was obtained for smeared polyelectrolytes 21,22,25] and is generalized here to annealed polyampholytes.

An instability of the homogeneous phase is determined by $S^{-1}\left(q_{0}\right)=0$, indicating a mesophase separation, where the size of the micro-domains is $\lambda_{0}=2 \pi / q_{0}$. In Fig. 3, the line marking the instability of the homogeneous (disordered) phase is plotted for different salt concentrations. At high ionic strength dilute polyelectrolyte solutions becomes unstable. This reflects the macroscopic phase separation of neutral (screened) polymers in a bad solvent $\left(q_{0} \rightarrow 0\right.$ as is seen in Fig. 2).

In Fig. 4 we show the dependence of the micro-domain region on the variance of the charge distribution, $\sigma^{2}$. One important consequence of eq. 60 is the equivalence of electrostatic screening induced by fluctuations in the polyampholyte charges and screening by added salt with effective bulk concentration $c_{\mathrm{b}}^{\mathrm{eff}}=\frac{1}{2} \sigma^{2} \phi_{\mathrm{b}}^{2}$. For example, for

$\phi_{\mathrm{b}}^{2}=10^{-6} \AA^{-3}, c_{\mathrm{b}}^{\text {eff }} / \sigma^{2}=0.8 \mathrm{mM}$. This effect is stronger in polyampholytes than in annealed polyelectrolytes, since in almost symmetric polyampholytes the excess charge is small, $|\bar{z}| \ll 1$, while the variance of the charges $\sigma^{2}$ can be close to one.

It is instructive to look at the instability of the homogeneous system towards macro-phase separation as a function of the polyampholyte average net charge. We emphasize that in some cases this macro-phase separation occurring at $q=0$ is preempted by a mesophase at $q_{0}>0$. Nevertheless, let us consider the change in the second virial coefficient $v \sim T-T_{\theta}^{0}$ due to the electrostatic interactions where $T_{\theta}^{0}$ is the $\theta$-temperature in the absence of electrostatic interactions. We note that for $\bar{z}=0$ there is no electrostatic contribution to $S(q)$ in our RPA calculation. For $\bar{z} \neq 0$, the $q=0$ instability will occur for

$$
|v| \phi_{\mathrm{b}}^{2}=\frac{\bar{z}^{2} \kappa_{p}^{2}}{\kappa_{s}^{2}+\left(|\bar{z}|+\sigma^{2}\right) \kappa_{p}^{2}}
$$

For fully charged polyampholytes (taking the bimodal distribution: $z_{j}= \pm 1$ and $|\bar{z}| \leq 1$ ), the $\theta$-temperature in the presence of charged monomers is

$$
T_{\theta}^{0}-T_{\theta}(\bar{z}) \sim \frac{\bar{z}^{2}}{2 c_{\mathrm{b}} / \phi_{\mathrm{b}}^{2}+|\bar{z}|+1-\bar{z}^{2}}
$$

This behavior is similar to the one found for single chains [7]. In Fig. 5 we plot the dependence of the $\theta$-temperature on the net charge $\bar{z}$ for different salt concentrations. As expected, the $\theta$-temperature is a decreasing function of the net charge $|\bar{z}|$. As can be seen from Fig. 5, addition of salt extends the bad solvent regime to higher values of $|\bar{z}|$. At high salt concentrations, the electrostatic interactions are screened and $T_{\theta}(\bar{z})$ is very close to its pure value $T_{\theta}^{0}$.

\section{CONCLUSIONS}

In this work we have studied bulk properties of charged polymers in aqueous solutions in the presence of added salt. Starting from a path integral formalism which takes into account the chain connectivity, short range and electrostatic interactions we derived mean-field equations describing the behavior of polyelectrolytes and polyampholytes in solution. We compared several models for the statistical charge distribution corresponding to different experimental realizations. The simplest and most frequently used model is the smeared one where charges are uniformly distributed. The permuted model, where the charges are mobile along the chain, is found to be equivalent to the smeared model except for a constant shift in the monomer chemical potential. This shift has to be taken into account in titration experiments.

The annealed model was found to have a lower free energy than the smeared one. This is related to the additional degrees of freedom. The effective dissociation depends on the local electric potential. At thermodynamic equilibrium, 
the quenched case is found to be equivalent to the annealed one, as long as the system is in contact with an infinite reservoir (bulk) of polyelectrolyte chains.

Annealed polyampholytes are characterized by their net charge and variance. We find different behavior for symmetric polyampholytes (no net charge) as compared to asymmetric ones (closer to polyelectrolytes). At low electrostatic potentials, all the above mentioned models have the same limiting behavior.

The monomer-monomer structure factor $S(q)$ is calculated within the Random Phase Approximation for annealed polyelectrolytes and polyampholytes. The electrostatic screening depends not only on the salt and counterions but also on the variance of the annealed charge distribution.

A peak in $S(q)$ for polyampholytes at finite wavenumber is shown to appear at a high net charge and/or low variance indicating polyelectrolyte-like behavior. For bad solvent conditions, the variance enhances the tendency of the system to undergo a mesophase separation.

Finally, it is worth mentioning that the present study can be further extended to treat polyelectrolytes and polyampholytes in restricted geometries and close to charged surfaces [31,43,45,47]. In particular, it will be interesting to address the question of how the different charge distribution is coupled with the polymer adsorption onto a single surface and the forces exerted by polyelectrolytes and polyampholytes between planar, cylinders or spherical surfaces.

\section{Acknowledgments}

We would like to thank L. Auvray, N. Dan, H. Diamant, J. Israelachvili, Y. Kantor and S. Safran for useful discussions. Two of us (IB and DA) would like to thank the Service de Physique Théorique (CE-Saclay) and one of us (HO) the School of Physics and Astronomy (Tel Aviv University) for their warm hospitality. Partial support from the Israel Science Foundation founded by the Israel Academy of Sciences and Humanities - centers of Excellence Program and the U.S.-Israel Binational Foundation (B.S.F.) under grant No. 94-00291 gratefully acknowledged.

[1] Oosawa F., Polyelectrolytes; Marcel Dekker: New York, 1971.

[2] De Gennes P. G., Scaling Concepts in Polymer Physics; Cornell Univ.: Ithaca, 1979.

[3] Barrat J. L. and Joanny J. F., Adv. Chem. Phys. 1996, 94, 1.

[4] Cabane B., Wong K., Wang T. K., Lafuma F. and Duplessix R., Coll. Pol. Sci. 1988, 266, 101.

[5] Dickinson E. and Eriksson L., Adv. Coll. Interface Sci. 1991, 34, 1.

[6] Wittmer J., Johner A. and Joanny J. F., Europhys. Lett. 1993, 24, 263.

[7] Kantor Y., Kardar M. and Li H., Phys. Rev. E. 1994, 49, 1383; Kantor Y. and Kardar M., Europhys. Lett. $1994,28,169$.

[8] Dobrynin A. V. and Rubinstein M., J. Phys. II France 1995, 5, 677.

[9] Everaers R., Johner A. and Joanny J. F., Europhys. Lett. 1997, 37, 275.

[10] Ha B. Y. and Thirumalai D., J. Phys. II France 1997, 7, 887.

[11] Fuoss R. M., Katchalsky A. and Lifson S., Proc. Natl. Acad. Sci. USA 1951, 37, 579.

[12] Manning G. S., J. Chem. Phys. 1969, 51, 954.

[13] Odijk T., J. Polym. Sci. 1977, 15, 477.

[14] Skolnick J. and Fixman M., Macromolecules 1977, 10, 944.

[15] Li H. and Witten T., Macromolecules 1995, 28, 5921.

[16] de Gennes P. G., Pincus P., Valesco R. M. and Brochard F., J. Phys. 1976, 37, 1461.

[17] Pfeuty P., J. Phys. 1978, 39, C2-149.

[18] Stevens M. J. and Kremer K., J. Chem. Phys. 1995, 103, 1669; Micka U. and Kremer K., Phys. Rev. E. $1996,54,2653$.

[19] Odijk T., Macromolecules 1979, 12, 688.

[20] Dobrynin A. V., Colby R. H. and Rubinstein M., Macromolecules 1995, 28, 1859.

[21] Borue V. Yu. and Erukhimovich I. Ya., Macromolecules 1988, 21, 3240.

[22] Joanny, J. F. and Leibler L., J. Phys. (France) 1990, 51, 547.

[23] Raphael E. and Joanny J. F., Europhys. Lett. 1990, 13, 623.

[24] Brereton M. G. and Vilgis T. A., Macromolecules 1990, 23, 2044; Vilgis T. A. and Borsali R., Phys. Rev. A. 1991, 43, 6857; Brereton M. G. and Vilgis T. A., J. Phys. I (France) 1992, 2, 581.

[25] Dormidontova E. E., Erukhimovich I. Ya. and Khokhlov A. R., Macrom. Theory Simul. 1994, 3, 661.

[26] Donley J. P., Rudnick J. and Liu A. J., Macromolecules 1997, 30, 1188.

[27] Moussaid A., Schosseler F., Munch J. P. and Candau S. J., J. Phys. II (France) 1993, 3, 573.

[28] Förster S., Schmidt M. and Antonietti M. Polymer 1990, 31, 781.

[29] Cohen Stuart M. A., J. Phys. France 1988, 49, 1001. 
[30] Cohen Stuart M. A., Fleer G. J., Lyklema J., Norde W. and Scheutjens J. M. H. M., Adv. Coll. Interface Sci. 1991, 34, 477.

[31] Fleer G. J., Cohen Stuart M. A., Scheutjens J. M. H. M., Cosgrove T. and Vincent B., Polymers at Interfaces; Chapman \& Hall: London, 1993; chapter 11.

[32] Haynes C. A. and Norde W., Coll. Surf. B 1994, 2, 517.

[33] Luckham P. F. and Klein J., J. Chem. Soc. Faraday Trans. I 1984, 80, 865.

[34] Marra J. and Hair M. L., J. Phys. Chem. 1988, 92, 6044.

[35] Claesson P. M. and Ninham B. W., Langmuir 1992, 8, 1406; Claesson P. M., Dahlgren M. A. G. and Eriksson L., Coll. Surf. A. 1994, 93, 293; Dahlgren M. A. G., Langmuir 1994, 10, 1580.

[36] Bergeron V., Langevin D. and Asnacios A., Langmuir 1996, 12, 1550.

[37] Van der Schee H. A. and Lyklema J., J. Phys. Chem. 1984, 88, 6661.

[38] Papenhuijzen J., Van der Schee H. A. and Fleer G. J., J. Coll. Interface Sci. 1985, 104, 540.

[39] Evers O. A., Fleer G. J. Scheutjens J. M. H. M. and Lyklema J., J. Coll. Interface Sci. 1985, $111,446$.

[40] Van de Steeg H. G. M., Cohen Stuart M. A., de Keizer A. and Bijsterbosch B. H., Langmuir 1992, 8, 8.

[41] Böhmer M. R., Evers O. A. and Scheutjens J. M. H. M., Macromolecules 1990 23, 2288.

[42] Muthukumar M., J. Chem. Phys. 1987, 86, 7230.

[43] Varoqui R., Johner A. and Elaissari A., J. Chem. Phys. 1991, 94, 6873.

[44] Varoqui R., J. Phys. (France) II 1993, 3, 1097.

[45] Borukhov I., Andelman D. and Orland H., Europhys. Lett. 1995, 32, 499.

[46] Borukhov I., Andelman D. and Orland H., Macromolecules 1998, 31, 1665; J. Phys. Chem. B, submitted.

[47] Podgornik R., J. Phys. Chem. 1992, 96, 695.

[48] Edwards S. F., Proc. Phys. Soc. 1965, 85, 613; Edwards S. F., Proc. Phys. Soc. 1966, 88, 265.

[49] Wiegel F. W., Introduction to Path-Integral Methods in Physics and Polymer Science; World Scientific: Singapore, 1986.

[50] Ground state dominance holds as long as $N \Delta E \gg 1$ where $N$ is the polymerization index and $\Delta E$ is the gap between the ground state and the first excited state. The next correction to ground state dominance involving an antisymmetric wave-function was considered by van Opheusden J. H. J., J. Phys. A: Math. Gen. 1988, 21, 2739.

[51] Landau L. D., Lifshitz E. M. and Pitaevskii L. P., Electrodynamics of Continuous Media, 2nd Ed.; Pergamon: Oxford, 1984.

[52] Edwards S. F. and Anderson P. W., J. Phys. F 1975, 5, 965.

[53] Cates M. E. and Ball R. C., J. Phys. (France) 1988, 49, 2009. 


\section{FIGURE CAPTIONS}

Fig. 1: Schematic view of a polyelectrolyte solution. The monomer coordinates are $\mathbf{r}_{l}(s)$ where $l=1, \ldots, M$ labels the polymer chain and $s \in[0, N]$ is a continuous index along the chain. The small ion coordinates are $\mathbf{R}_{i}^{+}$and $\mathbf{R}_{j}^{-}$where $i=1, \ldots, N^{+}$and $j=1, \ldots, N^{-}$.

Fig. 2: The effect of salt concentration on the structure factor $S(q)$ of polyelectrolytes, eq. 57. The parameters used are: $\phi_{\mathrm{b}}^{2}=10^{-6} \AA^{-3}, a=5 \AA, v=0.1 a^{3}, \bar{z}=0.1$ and $\sigma^{2}=0$. The salt concentration is: $c_{\mathrm{b}}=1 \mathrm{mM}$ (solid curve); $c_{\mathrm{b}}=2 \mathrm{mM}$ (dots); $c_{\mathrm{b}}=5 \mathrm{mM}$ (short dashes) and $c_{\mathrm{b}}=10 \mathrm{mM}$ (long dashes). The inset shows the effect of the average monomer charge at small salt concentration $c_{\mathrm{b}}=0.1 \mathrm{mM}$. The different curves correspond to: $\bar{z}=0.10$ (solid curve); $\bar{z}=0.03$ (dots) and $\bar{z}=0.01$ (dashes).

Fig. 3: The effect of salt concentration on the stability line of the homogeneous phase. The parameters used are: $a=5 \AA, w=5 \times 10^{5} \AA^{6}, \bar{z}=0.01$ and $\sigma^{2}=0$. The salt concentration is: $c_{\mathrm{b}}=0$ (solid curve); $c_{\mathrm{b}}=1 \mathrm{mM}$ (short dashes); $c_{\mathrm{b}}=1.5 \mathrm{mM}$ (dots and short dashes); $c_{\mathrm{b}}=2.5 \mathrm{mM}$ (long dashes) and $c_{\mathrm{b}}=5 \mathrm{mM}$ (dots and long dashes).

Fig. 4: The effect of the variance $\sigma^{2}$ on the stability line of the homogeneous phase. The parameters used are: $a=5 \AA, w=5 \times 10^{5} \AA^{6}, \bar{z}=0.01$ and $c_{\mathrm{b}}=0.01 \mathrm{mM}$. The different curves correspond to $\sigma^{2}=0$ (solid curve); $\sigma^{2}=0.01$ (short dashes) and $\sigma^{2}=0.04$ (long dashes).

Fig. 5: The shift in the $\theta$-temperature of polyampholytes: $\Delta T_{\theta}=T_{\theta}-T_{\theta}^{0}$ in units of $T_{\theta}^{0}$ as function of the excess charge $|\bar{z}|$ for different salt concentrations, eq. 62. Only the fully charged polyampholyte case: $z_{j}= \pm 1$ is shown. The polymer concentration is $\phi_{\mathrm{b}}^{2}=10^{-6} \AA^{-3},|v| \phi_{\mathrm{b}}^{2}=0.01$ and the salt concentration: $c_{\mathrm{b}}=0$ (solid curve); $c_{\mathrm{b}}=10 \mathrm{mM}$ (dots); $c_{\mathrm{b}}=0.1 \mathrm{M}$ (dashes); and $c_{\mathrm{b}}=1 \mathrm{M}$ (long dashes). 
Fig. 1

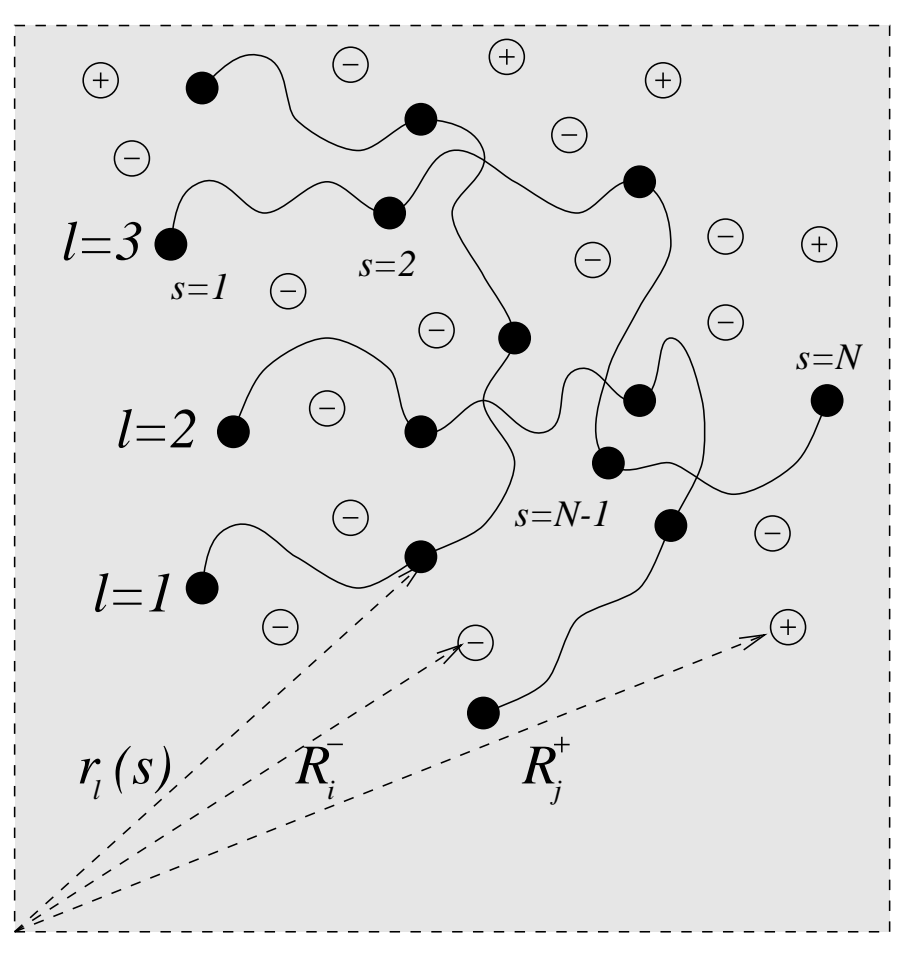

Fig. 2

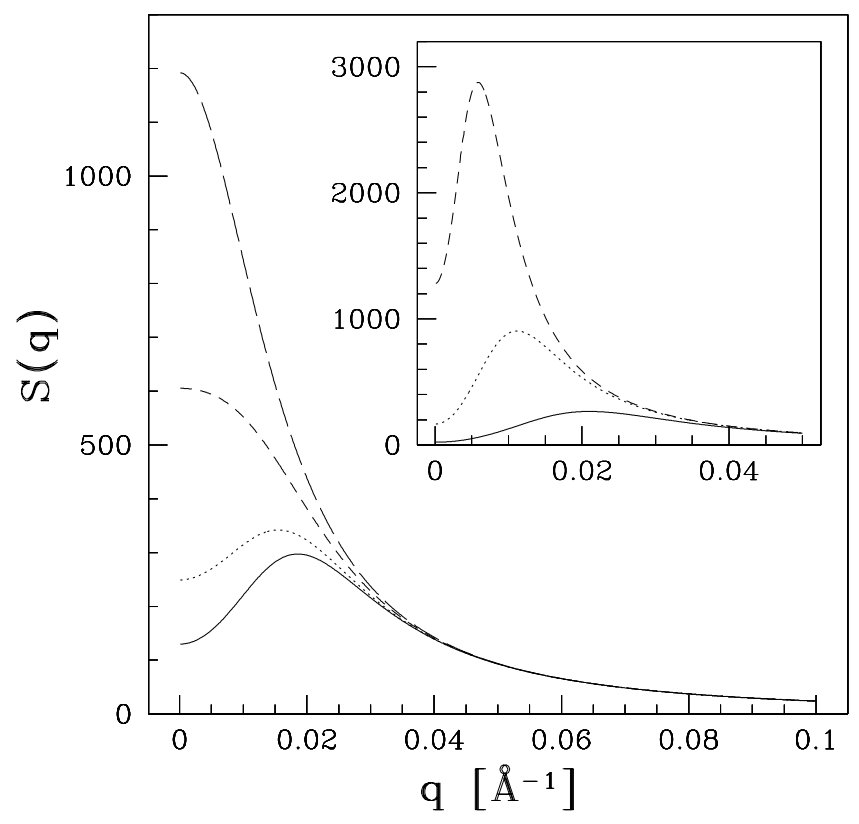


Fig. 3

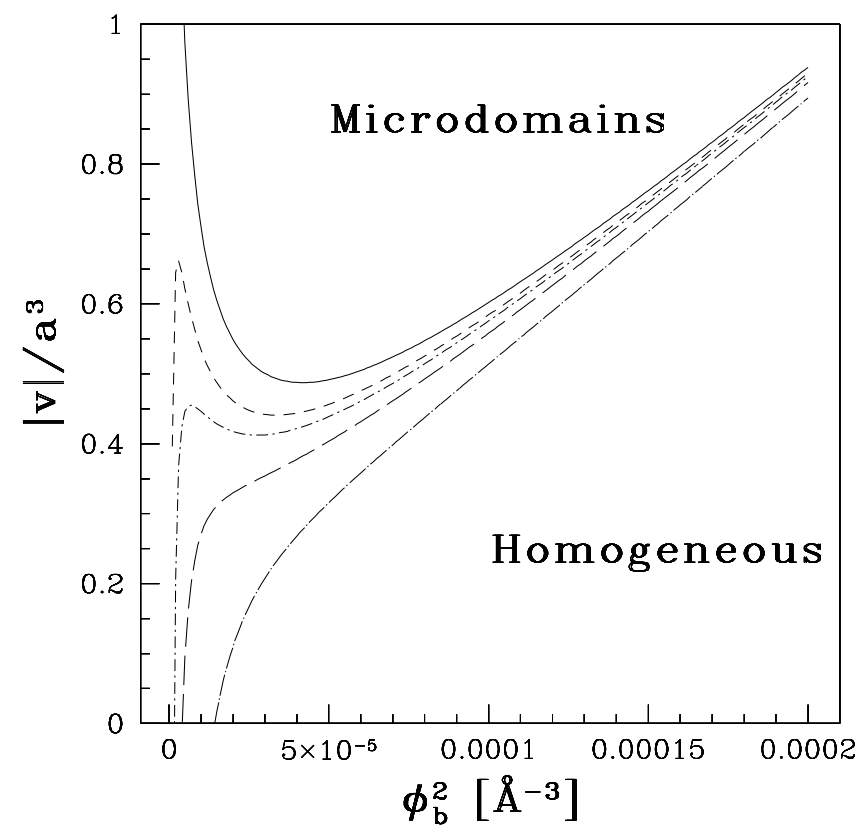

Fig. 4

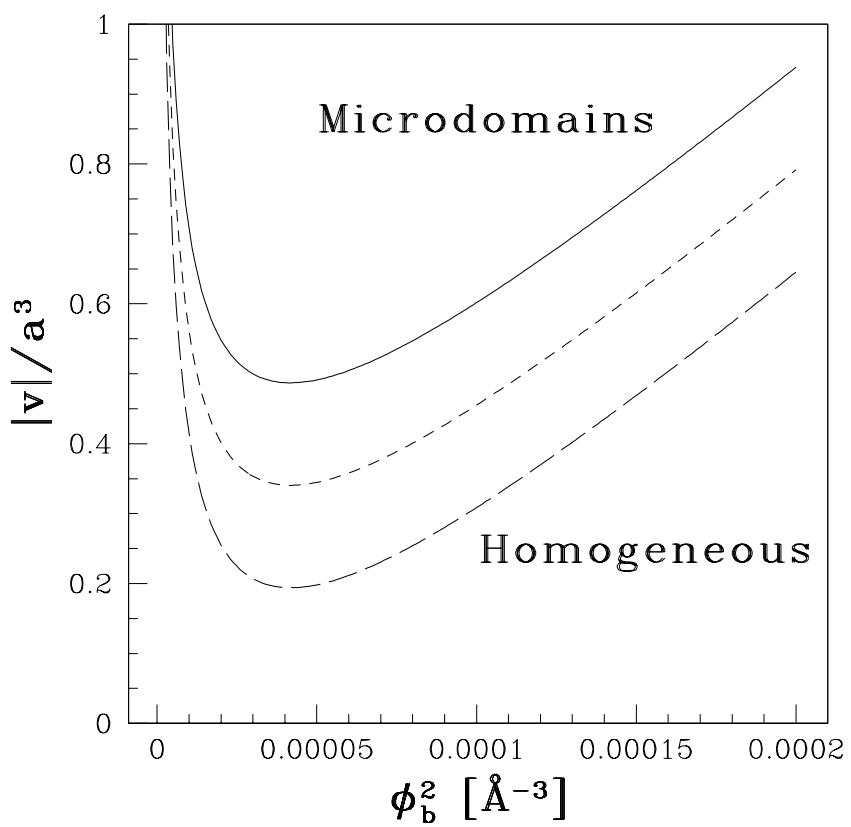


Fig. 5

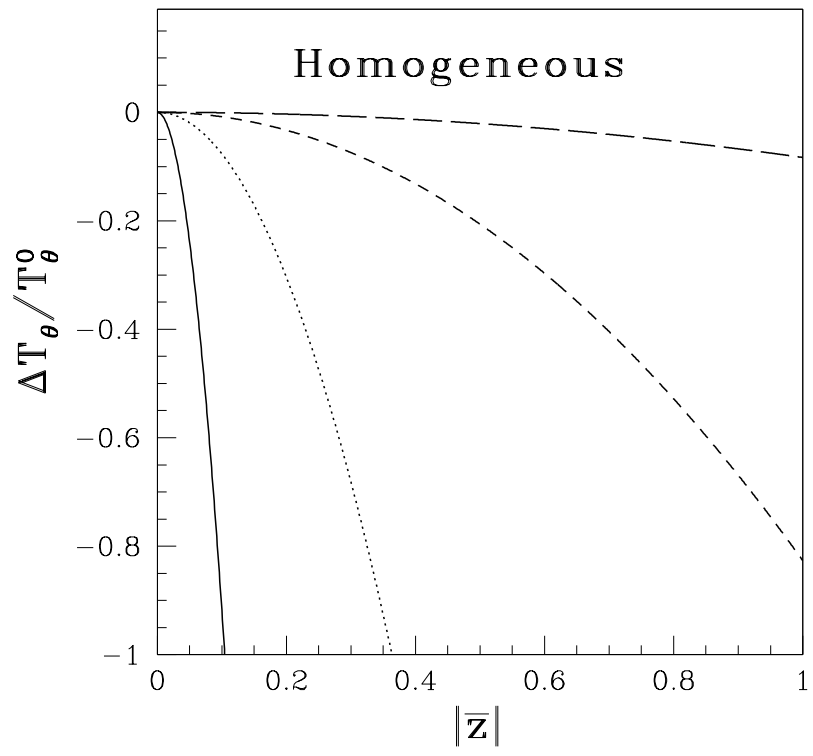

